Filigrane

Écoutes psychanalytiques

\title{
Des fourmis. En parlant du cadre
}

\section{Karine Roy-Déry}

Volume 24, numéro 1, printemps 2015

URI : https://id.erudit.org/iderudit/1033087ar

DOI : https://doi.org/10.7202/1033087ar

Aller au sommaire du numéro

\section{Éditeur(s)}

Revue Santé mentale au Québec

ISSN

1192-1412 (imprimé)

1911-4656 (numérique)

Découvrir la revue

Citer ce document

Roy-Déry, K. (2015). Des fourmis. En parlant du cadre. Filigrane, 24(1), 135-140. https://doi.org/10.7202/1033087ar d'utilisation que vous pouvez consulter en ligne.

https://apropos.erudit.org/fr/usagers/politique-dutilisation/ 


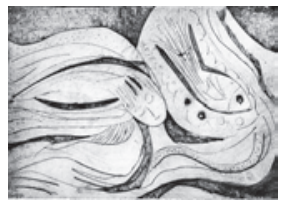

\title{
Des fourmis. En parlant du cadre
}

\author{
Karine Roy-Déry
}

\begin{abstract}
Si un adulte prétendait nous faire accepter l'objectivité de ses phénomènes subjectifs, nous verrions dans cette présentation la marque de la folie. Toutefois, si l'adulte parvient à jouir de son aire personnelle intermédiaire sans rien revendiquer, il n'est pas exclu que nous puissions y reconnaître nos propres aires intermédiaires correspondantes.
\end{abstract}

Winnicott, Jeu et réalité, 1971

\section{Tout d'abord...}

ans la même semaine, des fourmis ont envahi mes deux bureaux. Dans un, ce sont de grosses fourmis charpentières qui ont tracé leur autoroute entre moi et le client; dans l'autre, de petites curieuses qui prennent possession de tout l'espace, explorant le sol, les murs, les fenêtres, les fauteuils et même nos pieds. Cette situation me rend folle. J'en deviens obsédée. Je ne suis plus disponible: j'observe les fourmis. Elles se sont introduites dans mon bureau à l'improviste, sans prendre de rendez-vous. Elles ne respectent aucun horaire et paient encore moins d'honoraires. Elles me dérangent et dérangent mes clients. Je n'ai plus d'espace à penser, je ne peux plus m'absenter: j'observe les fourmis. Elles se sont même introduites dans le discours: les clients me parlent des fourmis, m'expliquent comment m'en débarrasser; bref, on jase fourmis. Certains tentent bien de faire comme si de rien n'était, mais sans vraiment y arriver. Les fourmis sont au centre du terrain de jeu, le jeu est paralysé... Et pourtant, une jeune fille a tenté de les rassembler dans une maison en carton: on ramenait les fourmis à la maison et les obligeait à y rester. Une dame a entrepris d'écraser toutes celles à sa portée, elle en a fait un petit tas, à ses pieds. Avec une ado, on a passé un long moment à en regarder une agoniser.... (J'avais mis du produit.) 


\section{Alors...}

J'aurais pu élaborer longuement sur le sens qu'ont pu prendre ces fourmis pour de nombreux clients. Comment ils ont ressenti cette présence, selon leurs enjeux respectifs. Mais, $j$ 'aurais davantage envie de vous parler du sentiment que j'ai ressenti d'une brèche (Bleger, 1966) dans mon cadre thérapeutique et de ma réaction à cette brèche. De mon contre-transfert, mais à l'égard de ces fourmis qui, indépendamment de la particularité de chaque client, ont mis en lumière la question de l'importance que le psychothérapeute accorde à son cadre. Du sens que, moi, j'accorde à mon cadre.

Le problème que je désire approfondir concerne les analyses où le cadre ne fait pas problème - dans le but, très précisément, de montrer qu'il est un problème - un problème qui n’a cependant été jusqu'ici ni défini ni reconnu. (Bleger, 1966)

Dans ce cas précis, le cadre n'a pas tant fait problème pour le client, mais bien pour le psychothérapeute. En effet, une invasion de fourmis, c'est beaucoup trop de réalité pour moi. Je ne suis pas une intervenante de terrain. Je veux fournir à mes clients un endroit neutre, protégé. J’y consacre beaucoup d'énergie. Je tente de rendre la température du local confortable, les locaux sont bien insonorisés (bon, il y a bien le son d'une ambulance à l'occasion mais vraiment pas trop souvent), je ferme la toile si le soleil est trop aveuglant, les fauteuils sont confortables et il y a même un ou deux coussins au besoin. En anglais, on dit «cosy». Je veux que le client ait toute la disponibilité pour être en contact avec ce qui se passe en lui, sans distractions.

[...] s'ajoute le dispositif matériel qui tempère les excitations physiques externes: bruit lumière, température, etc., ce qui permet à la séance de se dérouler dans une niche écologique relativement protégée. Le cadre psychanalytique minimise les stimulations exogènes et maximise l'attention portée à l'excitation interne, première condition de sa compréhension (Anzieu, 2003, 386)

Les fourmis viennent tout saccager. Mon oasis de paix et de tranquillité, ma niche écologique (Anzieu, 2003). Elles me font réaliser que moi aussi j'ai besoin d'un endroit neutre, protégé. J'ai besoin de me sentir en sécurité pour pouvoir accueillir toute cette peine, toute cette souffrance, ces traumas et cette intensité qui se promènent dans mon bureau à intervalle 
régulier. Cette régularité, ce rythme, cette organisation, ce "continuum» (Roussillon, 2007) que j'ai construit avec le temps sans trop m'en rendre compte est aujourd'hui bousculé. Les fourmis ont leurs règles à elles; elles ne connaissent pas les miennes. Elles ne semblent même pas s'en soucier: je n'existe pas.

Winnicott aborde aussi les cas où la tâche d'être mère ne peut être assurée correctement. Il décrit comment les carences maternelles interrompent le «continuum» de l'enfant et peuvent engendrer une «menace d'annihilation », laquelle correspond à une angoisse primitive très réelle qui met en péril l'existence même du self. (Roussillon, 2007, 68)

Intéressant, cette angoisse ressentie face à un être qui nous renvoie le sentiment de ne pas exister. Cette panique, cet état, je l'ai rencontré chez beaucoup de clients. Si l'autre ne me considère pas, je n'existe pas. Alors, on travaille vraiment très fort pour obtenir de la considération. On en fait trop; on travaille fort pour exister dans le regard de l'autre. Souvent, ça ne donne pas grand-chose. Et on s'y accroche. Avec les fourmis, j'entre en contact avec cette partie de moi, qui a besoin de considération, besoin d'être respectée. Je suis sensible à l'intrusion. Je sens l'intensité de ma réaction. Il se passe quelque chose. Je touche à une partie sensible de mon être. Je deviens le patient.

J'aime beaucoup les théoriciens qui voient le développement non pas comme une ligne droite, mais comme la coexistence de différents niveaux de développement. Qui pensent que nous avons tous, en nous, des aspects plus régressifs à côté d'autres plus évolués.

Il est possible que le moi dit pleinement développé, le moi qui aurait atteint sa pleine maturité, ne soit pas celui qui se serait fixé au stade présumément le plus élevé ou le plus avancé du développement, ayant laissé les autres stades derrière lui, mais plutôt celui qui intègre sa réalité de telle sorte que les stades d'intégration moi-réalité plus primitifs et plus profonds demeurent vivants comme sources dynamiques d'une organisation plus élevée. (Loewald, 1951, 365)

Cette vision me rassure quand je croise, en moi, à la vue de ces fourmis, cette folle hystérique qui met du produit, passe ses intervalles entre les clients à traquer les bêtes en question pour les exterminer mais aussi, qui 
sursaute de dégoût quand vient le temps de ramasser les corps morts, et encore davantage quand une de ses envahisseuses, supposément décédée, a le malheur de bouger une patte (AAAAAHHHHH!).

C'est cette partie de moi qui me vient à l'esprit quand je lis Bleger et qu'il pose la question...

En ce qui concerne ce dernier [le cadre], l'alliance est établie avec la partie psychotique (ou symbiotique) de la personnalité du patient (la question de savoir s'il en est de même pour la partie correspondante de la personnalité du psychanalyste reste encore pour moi sans réponse) (Bleger, 1966)

Parce que ces fourmis m'amènent à penser que dans le cadre, il ne se dépose pas que les aspects psychotiques du client!

\section{Et puis...}

La question du cadre, elle n'est pas nouvelle. Bleger dit: «Le cadre, en fait, se réfère à une stratégie plutôt qu'à une technique.» (Bleger, 1966) Cette «stratégie» semble avoir pour but de favoriser, ou même de faire émerger un accès au monde interne. Cette stratégie que l'on développe en devenant psychothérapeute, et qui souvent, était là bien avant notre «formation». Cela me ramène à l'essence même de ce qui fait que l'on devient psychothérapeute. Doris-Louise Héneault a creusé cette question dans son texte «Faire le métier d'un Trop et d'un Pas assez»:

Dans cette étude, j'avancerai l'hypothèse qu'à l'origine de la vie psychique du futur thérapeute domine l'envahissement parental lié inéluctablement à une absence et que la conséquence de cette dualité est l'idéalisation de la vie, des émotions, des idées afin de se surélever au-delà des contingences de la vie. (Doris-Louise Haineault, 1993, p.69)

Cela m'amène à penser que l'idée de se surélever au-delà des contingences de la vie (et par conséquent, des fourmis) puisse être une stratégie, pour reprendre Bleger. Et que cette stratégie, cette façon de faire pour le psychothérapeute, et le cadre qui en découle, peuvent avoir été à l'origine une modalité défensive. La difficulté vient du fait que les contingences de la vie sont constamment présentes d'une façon ou d'une autre et font parfois même irruption, malgré nous, dans la situation analytique. 
C'est ainsi que nous sommes mieux à même de comprendre la situation catastrophique qui, à un degré ou à un autre, survient toujours lors de la rupture par l'analyste du cadre, c'est-à-dire lors des vacances, de modification d'horaire, etc. car ces ruptures sont les occasions de l'ouverture d'une «brèche» par laquelle s'infiltre une réalité qui apparaît au patient comme catastrophique. (Bleger, 1966)

L'intrusion ou la réalité peut aussi être perçue comme menaçante pour le psychothérapeute et le mettre en contact avec ses propres enjeux, avec la partie psychotique de son être. Cette partie qui a survécu au Trop et au Pas assez dont parle Héneault (1993) et qui a besoin de cette niche écologique (Anzieu) pour arriver à utiliser les mécanismes que le psychothérapeute a développés pour y faire face: l'espace transitionnel, le langage, l'absence, la symbolisation.

Le cadre a beaucoup été mis en lien avec le holding de la mère. Je le vois bien quand je réfléchis à mon cadre, à ma conception de mon cadre: chaleur, contenance, pare-excitation. Roussillon décrit les besoins de la mère pour arriver à fournir ce «holding»:

On conçoit que cette période soit une période de «vulnérabilité» psychique particulière de la mère, une période de «transparence» psychique comme l'écrit M. Bydlowski (1978), et qu'elle ait besoin elle-même d'un environnement suffisamment protecteur. (Roussillon, 2007, 59)

Je reformulerais: Le psychothérapeute a besoin d'un environnement suffisamment protégé pour offrir un cadre suffisamment bon. "Le cadre ne prend sens qu'à partir du moment où il devient approprié à ce pourquoi il est construit.» (Geissmann-Chambon, 2003, 359) Et, selon moi, le cadre est au service du psychothérapeute autant que du client. Il est nécessaire pour arriver à contenir nos propres aspects psychotiques.

Les fourmis ont attaqué mon cadre et amené de nombreux bouleversements: intrusion, envahissement, réalité, motricité, impuissance, contact physique. Elles ont transgressé à elles seules de nombreux aspects fondamentaux de mon cadre implicite. Tout cela sans aucune considération à mon égard. Je ne pouvais même pas m'en défendre en interprétant leurs mouvements. En effet, le psychisme des fourmis m'est étranger...

Je me suis donc retrouvée seule avec ma propre folie.

Je suis folle. Je le sais. J'ai cette facilité à entrer en contact avec un éventail impressionnant d'émotions. Cette capacité à être vraiment là, dans 
l'émotion. À me laisser envahir. Je pense que c'est ce qui me permet de pouvoir être avec le client. Que ces aspects régressés et infantiles, ils ne sont pas si loin, en moi. Je les porte. J'ai beaucoup de tendresse pour ces délires immatures, ces fantaisies rocambolesques, ces impulsions irrationnelles. J'en suis toute pleine. Bon, j'avoue ce n'est pas toujours facile et je ne suis pas exemptée de conflits internes. Mais j'aime penser que d'une certaine façon, il existe en moi un Happy Kingdom ${ }^{1}$, qui peut soutenir, tolérer et même embrasser la possibilité de la survenue d'un conflit inconscient. Un Happy Kingdom, curieux, qui peut se faire surprendre et déstabiliser.

Même par de toutes petites fourmis...

Tout cela dans le cadre rassurant de mon bureau, suffisamment surélevé au-delà des contingences de la vie (Haineault, 1993).

\author{
Karine Roy-Déry \\ 960, rue Jean-Talon Est, bureau 4, \\ Montréal, Québec, H2R 1V4 \\ k.roydery@gmail.com
}

\title{
Notes
}

1. Terme utilisé par le $\mathrm{D}^{\mathrm{r}}$ Charles Levine pour expliciter les théories de Loewald lors d'une conférence conjointe de la Société canadienne de psychanalyse et la Société psychanalytique de Montréal, 2013.

\section{Références}

Anzieu, D. (2003). Cadre psychanalytique et enveloppes psychiques. Dans C. Geissmann et D. Houzel (dir.), Psychothérapies de l'enfant et de l'adolescent (p. 385-394). Paris: Bayard. Bleger, J. (1966). Psychanalyse du cadre psychanalytique. Dans D. Anzieu, R. Kaës et al. (dir.) Crise, rupture et dépassement. Paris: Dunod, 1979. http://www.psychanalyse.lu/articles/ BlegerPsychanalyseCadre.htm

Geissmann-Chambon, C. (2003). Un cadre approprié. Dans C. Geissmann et D. Houzel (dir.), Psychothérapies de l'enfant et de l'adolescent (p. 359-374). Paris: Bayard.

Haineault, D.-L. (1993). Faire le métier d'un Trop et d'un Pas Assez. Filigrane, 2, 68-77. Loewald, H. (1951/2011). Moi et réalité, Revue française de psychanalyse, 75 (2), 349-365.

Roussillon, R. (2007). Manuel de psychologie et de psychopathologie clinique générale. Paris: Masson.

Winnicott, D.W. (1971). Jeu et réalité. Paris: Gallimard, 2004. 